\title{
Monte Carlo simulations of ion channeling in crystals containing dislocations and randomly displaced atoms
}

Przemyslaw Jozwik ${ }^{1, a)}$, Lech Nowicki², Renata Ratajczak², Anna Stonert ${ }^{2}$, Cyprian Mieszczynski², Andrzej Turos ${ }^{2,3}, K^{2}$ rysztof Morawiec ${ }^{4}$, Katharina Lorenz ${ }^{1,5}$, Eduardo Alves $^{1}$

1IPFN, Instituto Superior Técnico, Universidade de Lisboa, Estrada Nacional 10, 2695-066 Bobadela, Portugal

${ }^{2}$ National Centre for Nuclear Research, A. Soltana 7, 05-400 Otwock-Swierk, Poland 3Institute of Electronic Materials Technology, Wolczynska 133, 01-919 Warsaw, Poland

${ }^{4}$ Institute of Physics, PAS, al. Lotników 32/46, 02-668 Warsaw, Poland

5Instituto de Engenharia de Sistemas de Computadores-Microsistemas e Nanotecnologias (INESC-MN), Rua Alves Redol 9, 1000-029 Lisboa, Portugal

\section{Keywords}

Monte Carlo simulation; Ion channeling; Defect analysis; Edge dislocation; Implantation/irradiation;

a) Corresponding author: p.jozwik@ctn.tecnico.ulisboa.pt; on leave from Institute of Electronic Materials Technology, Warsaw, Poland 


\begin{abstract}
Monte Carlo (MC) simulations are a powerful tool for the analysis of ion-solid interactions. The MC code McChasy (Monte Carlo CHAnneling SYmulation) allows the evaluation of Rutherford Backscattering Spectrometry spectra in the Channeling mode to quantify implantation damage. The code works on common PCs and takes into account randomly displaced atoms as well as certain types of extended defects. In this paper, we report recent improvements of the McChasy code, including a unique approach to the calculation of impact parameters between ions and target atoms in three dimensions (along with computing thermal vibrations also in 3D). Furthermore, the use of a rotation matrix to provide different orientations of dislocation lines and an updated model of edge dislocations were also implemented in the code. Dislocation parameters are obtained directly from high-resolution Transmission Electron Microscopy micrographs. Two case studies are presented to highlight the importance of these improvements: Ni-implanted $\mathrm{Al}$ was analyzed as an example of a crystal mainly containing dislocations; Er-implanted $\mathrm{ZnO}$ was studied, revealing the strength of MC analysis for materials containing a mixture of different defect types, namely randomly displaced atoms and dislocations.
\end{abstract}

\title{
I. INTRODUCTION
}

Analysis of lattice disorder in crystals is of high importance in materials science, especially for structures intentionally modified by ion beams or unintentionally exposed to radiation. Materials processing, e.g. for application in electronics, often uses ion beams for doping to achieve the desired properties. However, since the interaction between ions and target atoms is a ballistic process it always leads to the formation of a large number of point defects, which can then interact to form complex defect structures. These may contain point defects, point defect clusters or amorphous regions, which have in common that they normally lead to a 
random displacement of atoms from their original lattice sites. However, in many crystals point defects can arrange in an ordered way to form extended defects, such as stacking faults and dislocation loops. Besides, ion implantation can cause compositional changes, in particular in compound hosts, voids, or lead to intermixing in layered structures.

One of the principal methods used in the analysis of lattice distortions in monocrystalline structures is Rutherford Backscattering Spectrometry in Channeling mode (RBS/C). It has been used to study the radiation damage in many materials for more than five decades. ${ }^{1}$ Beams consisting of light ions (e.g. $\left.\mathrm{H}^{+}, \mathrm{He}^{+}\right)$can penetrate a crystal along channels formed by atomic rows due to the proper alignment between the beam and one of the crystallographic directions. In such conditions, the measured backscattering yield is very sensitive to the presence of defects.

Evaluation of the RBS/C spectra of defect-containing crystals is commonly based on the Two-Beam Approximation (TBA). ${ }^{2}$ The method assumes that in the presence of defects the measured backscattering yield increases due to two mechanisms: direct backscattering of ions from displaced atoms and dechanneling of the beam due to small-angle scattering leading to enhanced backscattering in the deeper regions of the crystal. However, different types of defects contribute differently to each of these mechanisms; in particular, dislocations hardly contribute to direct scattering and mostly interact with the beam by dechanneling, while randomly displaced atoms contribute to both mechanisms. ${ }^{3}$ The fraction of the direct backscattering and the dechanneling to the measured yield can be evaluated analytically, e.g. using iterative procedures shown by Jin et al. ${ }^{4}$ However, the method does not allow extracting the exact concentration-profiles of the different defect types and derives only a level of relative disorder.

Quantitative separation of different types of defects is a hard task, indeed. Simplified evaluation of RBS/C spectra assumes e.g. the presence of point defects only and neglects the 
contribution to dechanneling from dislocations or dislocation loops. Therefore, the results may be misleading or incomplete. To extract quantitative information about lattice distortion from RBS/C spectra, computer simulations can be employed based on the Monte Carlo (MC) approach, which can be supported by Molecular Dynamics (MD). Many MC techniques are based on rather small virtual structures (with sizes of up to a few tens of lattice constants) that can be modified during the simulation process according to the model of the defects used in the code. In this approach implementation of different types of defects is strongly limited. MD enables the creation of large structures containing a variety of defect types; however, it requires considerable amounts of computing time and memory.

To date, several codes have been developed to analyze channeling data. ${ }^{5-14}$ Most of them ${ }^{5-12}$ can consider only simple defects. However, Zhang et al. proved that RBS/C spectra are highly sensitive to extended defects, ${ }^{14}$ so their presence should not be neglected. However, their code uses very large cells, which have to be customized for each sample in the MD simulations. Therefore, analysis and simulations are highly time- and computer memoryconsuming. The separation of linear defects (dislocations) from randomly displaced atoms can be achieved by the McChasy (Monte Carlo CHAnneling SYmulation*) code. The great virtue of the code is also the possibility of running it on common PCs. ${ }^{13}$

In this paper, further developments of the dislocation model, as well as additional improvements applied to the code, are described. The theory section reviews the main principles of the MC engine used in the McChasy code and describes its newest features. The experimental section describes a new approach to the analysis of high-resolution Transmission Electron Microscopy (HRTEM) micrographs to incorporate a realistic description of the lattice distortion into the dislocation model applied. The last three sections

\footnotetext{
* The letter ' $\mathrm{Y}$ ' comes from the Polish translation of the word 'simulation', which is 'symulacja'. 
are devoted to selected case studies using the McChasy code, discussion of the results and the most important conclusions.

\section{THEORY}

\section{A. Monte Carlo simulations for ion channeling}

MC simulations are a computational method of reproducing ion movement in crystals and, as a result, they can provide a simulated RBS spectrum to fit experimental data. Inspired by the

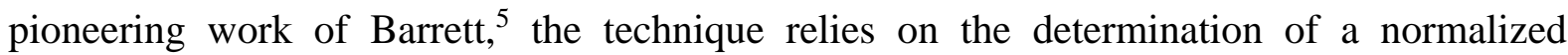
nuclear encounter probability (NEP). For each ion-atom interaction, the probability of scattering is calculated and stored in memory and eventually converted into an RBS spectrum. The use of the NEP also allows the number of calculated trajectories sufficient to provide acceptable statistics to be reduced. This approach is used in the MC engine of the McChasy code.

During the simulation process, the ion trajectories are computed by a series of iterations. Each iteration is associated with the determination of ion deflections, which must be calculated taking into account the screened Coulomb potential, thermal vibrations of atoms and the energy loss within a depth interval in the crystal. MC codes can differ from each other concerning the choice of these parameters as well as in the way they compute the ionatom interactions. The most commonly used method for this purpose is called the Binary Collision Approximation (BCA). ${ }^{15}$ In this approach, the ion interacts only with one atom at a time. Its deflection angle is calculated based on the impact parameter. Between collisions, the ion moves along a straight path.

In many codes, only the interaction with the nearest atom is considered and the presence of more distant atoms is neglected. A method, used in the McChasy code, to compute the influence of more than one atom on the trajectories of the channeled ions is called the Planar 
Scattering Approximation (PSA). In this model, a channeled ion interacts simultaneously with several atoms located on a plane that is perpendicular to the channeling direction. Such a monolayer is also called a slice of atoms. Between atomic slices, the ion moves along a straight path. ${ }^{3}$ This approach allows determination of ion deflections not only for the nearest neighbor; atoms that are more distant can also be taken into account, depending on their impact parameters.

However, in this method, the impact parameters are calculated within the plane of a given atomic slice as the distance between a given atom and the impact point of the channeled ion on the plane (i.e. always perpendicular to the channeling axis). Therefore, while calculating the impact parameter, the real direction of the ion between the slices is in fact neglected.

More details about the preparation of sliced structures and the computation of deflections are provided in §II.B. A new 3D model for impact parameter calculations within the PSA approach is described in §II.C.1.

\section{B. The McChasy code - general features}

The McChasy code is based on the computational techniques described in §ll.A. Its current state is a further development of the versions reported previously. ${ }^{8,11,13}$ The most important features of the code may be summarized as:

(i) The McChasy code uses the PSA approach to calculate the interactions of channeled ions with target atoms. Therefore, the structure read by the code must be initially prepared by an associated code called Structure Preparation. Based on the space group and the unit cell of the chosen structure, the code calculates the coordinates of atoms and groups them into monolayers (atomic slices) that are orthogonal to a chosen crystallographic direction $z$. All atoms within a single slice have the same coordinate $z$. The lateral dimensions of the slices usually do not exceed a few lattice spacings. The McChasy code uses such a sliced cell structure 
and repeats it periodically during the simulations. An example of a structure created by the Structure Preparation code for [011] Al is shown in FIG. 1. The structure belongs to the Fm-3m space group.

A parallelogram is defined in the center of the atomic slices, which is built based on the $x$ and $y$ coordinates of the translation vectors. The parallelograms on the various planes determine a virtual channel for calculations of ion trajectories (cf. FIG. 1). When the simulations begin the initial position of every ion is picked randomly inside the parallelogram of the first layer (the surface) of the chosen structure. The deflection angle and the impact point of the ion on the subsequent atomic slice are determined according to the procedure described in (v). Although the ion can interact with all atoms located in the given slice (depending on the impact parameter), it is ensured that its position always remains inside the parallelogram. If any ion should leave the virtual channel it is returned to the equivalent position inside the parallelogram using the appropriate translation vectors. This procedure allows computer memory to be saved since it uses virtual structures of relatively small size.

FIG. 1. Schematic Al structure used in the McChasy code for the [011] channeling direction: (a) projection along the [011] direction showing the top two slices of atoms; (b) side view of all four atomic slices describing the structure. The distance between the slices (in the $z$-direction) is 143.17 $\mathrm{pm}$. The planar spacings for the $x$ and $y$ directions are $404.95 \mathrm{pm}$ and $572.69 \mathrm{pm}$, respectively. A virtual parallelogram in the center of each slice is marked with a dashed black line.

(iii) Thermal vibrations of target atoms are treated as random displacements determined from an implemented Gaussian distribution ${ }^{16}$. Their amplitude can be adjusted before the simulations.

(iv) The energy loss is calculated allowing a choice between two tables of the stopping power $\left(S_{r}\right)$ : calculated by the SRIM code (default) ${ }^{17}$ or by the RUMP code. ${ }^{18}$ However, the energy loss of channeled ions is lower and depends on the 
ion impact parameter $b$. Channeling stopping power $\left(S_{c h}\right)$ in the McChasy code is computed in terms of the classical Lindhard approach ${ }^{19}$ according to the formula:

$S_{c h}=(1-\propto) S_{r}+\propto S_{l}(b)$,

where $S_{l}(b)$ is the local stopping power calculated based on $S_{r}$ and the local densities of electrons (both valence and core) $)^{8,20}$. An adjustable parameter $\alpha$ is by default set equal to 0.5 for the channeling condition and 0 for the random case. However, its exact value may vary depending on the structure. ${ }^{21}$ Possible uncertainty of the depth conversion does not exceed $30 \mathrm{~nm}$ as evaluated at the depth of $100 \mathrm{~nm}$ and $200 \mathrm{~nm}$ during $\mathrm{MC}$ simulations of $1.7 \mathrm{MeV} \mathrm{He}$ ions channeling in $\mathrm{ZnO}$ using the most extreme mismatch of the value of $\alpha(\alpha=1$ and $\alpha=0$ taken instead of $\alpha=0.5$ ).

The energy loss is applied to channeled ions at every slice of atoms after the calculation of the interaction with the atoms.

(v) The deflection angles are calculated within the framework of the $B C A$ in two ways: for small deflections (e.g. below $0.000235^{\circ}$ for $\mathrm{Li}$ and below $0.037^{\circ}$ for $\mathrm{U}$ ) an analytical approximation to the equations of motion based on the Gauss-Mehler quadratures is applied; ${ }^{22,23}$ for larger deflections numerical integration of the equations of motion based on the Euler-Cromer method is used.24,25 Both computational methods use the Ziegler, Biersack, Littmark (ZBL) universal screened potential. ${ }^{17}$ The values of the deflection angles are tabulated for 90 elements from $\mathrm{Li}$ to $\mathrm{U}$ as a function of the impact parameter. During the simulation, the McChasy code calculates the impact parameters of a channeled ion relative to all atoms on a current slice (after thermal vibrations are applied). If the impact parameter is below a previously defined limit (different for each element), the code reads the corresponding deflection angles from the table, while collisions 
with very large impact parameters are not taken into account. Once the resulting deflection angle summing over all interacting atoms is calculated, it is applied to determine the new ion momentum. Subsequently, the point of impact on the following slice of atoms is determined.

(vi) Multiple scattering on electrons can be applied optionally according to the Lindhard approach ${ }^{19}$ as an additional change in the ion direction after a single collision with a target atom. The new direction is calculated using a Gaussian distribution with dispersion parameter $\sigma^{2}$ depending on the energy of the ion before the collision and its energy loss. However, the influence of this effect on the simulated spectra is negligible. ${ }^{8}$

(vii) Point defects may be considered during the simulation process in three ways:

- Substitutions: target atoms are randomly substituted by a chosen impurity element with a probability calculated from a depth-profile postulated by the user.

- Vacancies: a target atom can be removed leaving a vacancy. For this purpose, a special element called Vo with no mass and no charge was introduced for use in the substitution procedure.

- Interstitials: target atoms are randomly shifted to new positions calculated before the impact parameters of a channeled ion are determined. The percentage of displaced atoms in each atomic slice depends on a depthdistribution provided as input data. Four types of point defect can be considered: Randomly Displaced Atoms (RDA), whose atomic displacements are calculated randomly over the atomic slice, are used by default; however, atoms can also be tossed around their positions with a Gaussian distribution or 
(with uniform probability) over a disk or a ring. ${ }^{11}$ This procedure also applies to substitutional atoms, if defined.

It should be noted that the possible charge and the lattice distortion around a point defect (vacancy or interstitial) are not taken into account in the defect models used by McChasy. The additional charge around the point defect has a negligible effect on the motion of channeled particles. ${ }^{8}$ The relaxation of the lattice around a point defect will lead to a displacement of the surrounding atoms from their lattice sites and, therefore, will increase the fraction of interstitial-type defects. For silicon, it was shown using computer simulations that certain point defects lead to the displacement of up to 9 atoms (9 displaced atoms were found for <110>-split interstitials and 4 for vacancies). ${ }^{26}$ In the case of GaAs, the number of displaced atoms per Frenkel pair was estimated experimentally to be $14 \pm 4$ in weakly damaged material. ${ }^{27}$

(viii) The presence of dislocations may be considered within the framework of the Peierls-Nabarro model of edge dislocations (DIS). ${ }^{28-30}$ Atomic planes adjacent to an extra half-plane introduced into the lattice and forming the dislocation are bent; the bending can be described by an arctan function. During the simulation process, the displacements of atoms in the vicinity of dislocations are calculated according to the equation:

$u(z)=d \arctan (g z)$,

where $z$ is the distance from the dislocation line in the channeling direction and $d$ and $g$ are fitting parameters (also called dislocation parameters). The factor $d$ is related to the distance between the function asymptotes $(D)$ by the dependency:

$d=D / \pi$ 
This parameter is therefore also closely associated with the Burgers vector of the dislocation. The parameter $g$ depends both on $d$ and the bending angle $\eta$, formed at the inflection point of the arctan function between a tangent to the function and a line parallel to the arctan asymptotes, according to the formula: ${ }^{31}$

$g=\tan \eta / d$.

Parameters $D$ and $\eta$ are also called the geometrical parameters of the dislocations. They both decrease for every adjacent atomic plane with the distance from the dislocation extra half-plane. The determination of their decay from the analysis of HRTEM micrographs is described in the experimental section.

To summarize, before the simulations start one needs to define some crucial input data:

(i) Parameters associated with the beam: energy $(0.8-3.5 \mathrm{MeV})$, element $\left({ }^{4} \mathrm{He},{ }^{3} \mathrm{He}\right.$, $\left.{ }^{1} \mathrm{H}\right)$, beam dispersion $\left(0.03^{\circ}\right.$ by default), straggling variance, and adjustment of a local energy loss factor.

(ii) Parameters of the experiment: scattering angle, the energy resolution of the detector, calibration of the energy channels width and offset.

(iii) Depth-distributions of defects (optional):

- $\quad$ point defects (interstitials and/or substitutions, including vacancies),

- edge dislocations (defined as an extra half-plane parallel to the channeling direction),

- stacking faults (defined as a lateral displacement between two following atomic slices orthogonal to the channeling direction),

- grain boundaries (defined as a lateral displacement and a tilt between two following atomic slices). 


\section{The McChasy code - improvements}

The McChasy code in its current state has been updated taking into account the following improvements:

\section{Three-dimensional (3D*) computing of the impact parameter}

In classic PSA, the thermal vibrations of target atoms are calculated separately for two dimensions $x$ and $y$ (orthogonal to the channeling direction $z$ ). Similarly, the impact parameters of ions are determined in 2 dimensions as distances between target atoms and the impact point of a channeled ion on the slice of atoms (cf. the PSA description in §II.A). Therefore, the real direction of the ion movement is used only to determine its position on the following slice and is neglected in the evaluation of the impact parameter.

From now on the McChasy code also calculates the third coordinate $z$ of the thermal vibrations. Moreover, the impact parameters of channeled ions are determined by taking into account the actual directions of the ions and atom positions evaluated in three dimensions (with 3D thermal vibrations applied).

In most cases, impact parameters calculated in $3 \mathrm{D}$ are smaller than those determined in $2 \mathrm{D}$ using the classic PSA. Therefore, the intensity of RBS spectra (simulated with all other conditions kept unchanged) is expected to be higher in the 3D than in the 2D approach. Simulations made for [0001] $\mathrm{ZnO}$ as presented in FIG. 2 confirm this assumption. Arbitrary symmetric distribution of RDA (with a maximum of $30 \%$ at a depth between 200-250 nm) was provided as input data and simulations using $2 \mathrm{MeV} \mathrm{He}^{+}$ions detected at $170^{\circ}$ were performed using the newest 3D approach and the previous 2D one. The differences between these two spectra become even more visible in the lower energy region corresponding to deeper regions of the crystal (behind the damage peak localized between $\sim 1.25-1.50 \mathrm{MeV}$ ) due to increasing dechanneling, which eventually intensifies scattering of ions.

\footnotetext{
* 3D can refer to 'three-dimensional' or 'three dimensions', depending on the context.
} 
FIG. 2. Simulated RBS/C spectra obtained by the MC code for $\mathrm{ZnO}$ with a $2.0 \mathrm{MeV} \mathrm{He}^{+}$beam along the [0001] direction detected at a backscattering angle of $170^{\circ}$. Arbitrary symmetric distribution of RDA (in-set) was postulated as input data for the simulations. The spectra were obtained using two models to calculate the impact parameters in the McChasy code: 3-dimensional (3D simulations) and 2dimensional (2D simulations).

\section{The improved model of dislocations}

The depth-distribution of DIS-type defects provided as input data is expressed in units of $\mathrm{cm}^{-}$

2. The McChasy code transforms the dislocation density into a certain number of dislocation lines, which are interpreted as dislocation edges. Only the lines intersecting a virtual cylinder built around the simulation channel (along the channeling direction) affect the ion movement and contribute to calculations of target atom displacements from Eq. (2). The radius of this cylinder (expressed as a multiple of the lattice spacing) is defined as input data.

Dislocation lines in McChasy can be imagined as distributed randomly over the simulated structure, based on their depth-distribution. Currently, the dislocation lines, as well as the Burgers vectors of dislocations, are perpendicular to the channeling direction. A rotation matrix was implemented for the first time to determine the orientation of the dislocations. So far only dislocation lines with low Miller indices have been tested: oriented in three main directions around the [0001] channeling direction in hexagonal structures (6-fold symmetry) and in two main directions around the [001] and the [011] channeling directions in cubic ones (4-fold symmetry). During the simulations, the arctan inflection point is assigned to the $z$ coordinate associated with a depth of a given dislocation line to calculate displacements of target atoms.

\section{Transparency effect}

The movement of ions in channels formed by surrounding atomic rows can be approximated as an oscillatory motion in the framework of the continuum-elastic model proposed by Lindhard. ${ }^{19}$ The component of the momentum perpendicular to the direction of motion 
undergoes harmonic changes due to the repulsive potential of the nuclei. However, in some circumstances, instead of being reflected, the ion can cross through the wall of surrounding atoms - the structure becomes locally transparent to the ions. This phenomenon is expected to occur more efficiently in the presence of dislocations due to the characteristic channel bending. ${ }^{32}$ By jumping to a neighboring channel the ion comes closer to or further from the dislocation half-plane. Therefore, it continues its motion in the new channel surrounded by less or more strongly bent atomic rows.

The new McChasy code is now modified taking into account this transparency effect. During the simulation process, the code tracks the distance between the virtual channel of ion motion and all the dislocation lines. If the ion leaves the virtual channel, as in the transparency effect, it is returned to the cell as described previously, but the distance to the appropriate dislocations is updated.

\section{EXPERIMENTAL DETAILS}

As mentioned in $\S \mathrm{B}$ (viii), the fitting parameters of the arctan function used in the model of dislocations (Eq. (2)) can be determined experimentally. The complementary technique used for this purpose is HRTEM. The results reported in our previous work were obtained using the Inverse Fourier Transform (IFT) of HRTEM micrographs. ${ }^{31,33}$ This method may, however, provide some misleading artifacts. Therefore, it is recommended to use the directly recorded HRTEM images instead.

Furthermore, to provide better precision the parameters $d$ and $g$ should be determined simultaneously during the analysis of the HRTEM data. In the studies reported in Refs. 31,33 they were calculated using Eqs. (3) and (4); however, the parameters $D$ and $\eta$ were determined separately using different software. From now on, simultaneous determination of the dislocation parameters is possible thanks to a script written for use in Microsoft Excel 
worksheets. ${ }^{34}$ By adjusting both parameters $d$ and $g$, bent atomic rows can be fitted by the arctan function as presented in FIG. 3. However, $d$ and $g$ have no obvious physical meaning, so it is possible to modify the parameters $D$ and the angle $\eta$ instead. The script converts them immediately into $d$ and $g$ (following Eqs. (3) and (4)). The script also works well for IFT of HRTEM micrographs, if desired.

This procedure was applied to recalculate previously obtained values of the dislocation parameters for $\mathrm{Al}_{0.4} \mathrm{Ga}_{0.6} \mathrm{~N}$ and $\mathrm{SrTiO}_{3}$ (using IFT of the HRTEM images) as reported in. ${ }^{31,33}$ Additionally, an HRTEM study was performed on ion-bombarded [0001] $\mathrm{ZnO}$ bulk single crystals provided commercially by MaTecK, Germany. The samples were bombarded at RT with $300 \mathrm{keV} \mathrm{Ar}^{+}$ions at the Institute of Electronic Materials Technology using the Balzers MBP 202RP ion implanter to fluences ranging from $1.0 \times 10^{13} \mathrm{~cm}^{-2}$ to $4.0 \times 10^{16} \mathrm{~cm}^{-2}$. To avoid sample overheating the current density of $\mathrm{Ar}$ ions was kept below $0.5 \mu \mathrm{Acm}^{-2}$. To avoid ion channeling the incident angle of the Ar beam was $\sim 7^{\circ}$. Cross-sectional HRTEM images were taken at the Institute of Physics of the Polish Academy of Sciences using a TITAN CUBED 80-300 TEM with a CCD camera operating in TEM bright field mode. The energy of the electron beam was $300 \mathrm{keV}$. Lamellas were prepared using a dual-beam Focused Ion Beam (FIB) with Ga and electron beams using a Pt mask. Samples bombarded with Ar ions above fluences of $5.0 \times 10^{14} \mathrm{~cm}^{-2}$ turned out too fragile and were destroyed during FIB preparation. Analysis of dislocation parameters was performed using the Microsoft Excel script. ${ }^{34} \mathrm{~A}$ selected HRTEM micrograph of Ar-bombarded $\mathrm{ZnO}$ is shown in FIG. 3. The solid red line refers to the arctan fit obtained using the script.

FIG. 3. Part of the cross-sectional HRTEM micrograph taken for a [0001] ZnO single crystal bombarded with 300-keV Ar ions to a fluence of $2.0 \times 10^{14} \mathrm{~cm}^{-2}$ with an edge dislocation visible. The green circle denotes the first atom of an extra-half plane of atoms. The red line is a fit to the bent atomic plane made using the Microsoft Excel script. ${ }^{34}$

Dislocation parameters $D$ and $\eta$ determined for $\mathrm{ZnO}$ and also reanalyzed for $\mathrm{SrTiO}_{3}$ (STO), and $\mathrm{AlGaN}$ reveal the following dependencies: 
$D(r)=D_{0} \exp \left(-\frac{r-1}{\delta}\right)$

$\eta(r)=\eta_{0} r^{-\varepsilon}$

where $\delta$ and $\varepsilon$ are decay parameters obtained from the analysis of the HRTEM data and $r$ is the distance from a dislocation edge expressed as a dimensionless multiple of the lattice constant. For example, $r=1$ denotes an atomic plane just next to an extra half-plane of a dislocation. The plane fitted with the red line in FIG. 3 is characterized by $r=2$. The parameters $D_{0}$ and $\eta_{0}$ refer to bent planes with $r=1$.

Selected examples of the decay of the parameters $D$ and $\eta$ determined for single dislocations revealed by HRTEM in all three analyzed structures are shown in FIG. 4.

FIG. 4. Dependence of the bending angle $\eta(a)$ and the distance $D$ between the arctan asymptotes (b) measured using the Microsoft Excel script for selected edge dislocations revealed by HRTEM for AIGaN, ZnO, and STO. Fits to the data series are plotted as dashed lines in the corresponding colors.

The parameters $D_{0}$ and $\eta_{0}$ as well as the decay parameters $\delta$ and $\varepsilon$ obtained in this research are shown in Table 1. The uncertainty is associated with the human ability to evaluate the precision of the fit to the bent atomic row seen in the image and it was estimated to be $3 \%$ of the obtained values.

\begin{tabular}{c|c|c|c|c} 
Structure & $D_{0}[\mathrm{pm}]$ & $\delta$ & $\eta_{0}\left[{ }^{\circ}\right]$ & $\varepsilon$ \\
\hline \hline $\mathrm{SrTiO}_{3}$ & $194 \pm 6$ & $42 \pm 2$ & $9.7 \pm 0.3$ & $0.96 \pm 0.03$ \\
$\mathrm{AlGaN}$ & $187 \pm 6$ & $7.7 \pm 0.3$ & $32 \pm 1$ & $0.91 \pm 0.03$ \\
$\mathrm{ZnO}$ & $166 \pm 5$ & $10.9 \pm 0.4$ & $60 \pm 2$ & $1.04 \pm 0.04$
\end{tabular}

Table 1. Fitting factors of the decay formulas of parameters $D$ and $\eta$ (Eqs. (5) and (6)) determined by the HRTEM analysis. Based on $D$ and $\eta$, the fitting parameters of the arctan function ( $d$ and $g$ ) are calculated to be used in the McChasy code as the model of dislocations.

\section{APPLICATION}


The new McChasy code, containing the features mentioned in the theory and experimental details sections, was subsequently used to analyze selected channeling spectra recorded for two structures: Ni-implanted $\mathrm{Al}$, containing mostly dislocations, and Er-implanted $\mathrm{ZnO}$, revealing the coexistence of both linear and point defects.

\section{A. Monte Carlo simulations for Ni-implanted Al}

FIG. 5 presents RBS spectra recorded for Al single crystals using 2.0 MeV He ions along the [110] direction detected at a backscattering angle of $160^{\circ}$, as reported by Picraux et al. in Ref. 35 and thoroughly discussed in Ref. 3 . The aligned and random spectra refer to a sample implanted at $\mathrm{RT}$ with $150 \mathrm{keV} \mathrm{Ni}$ ions. The spectra were digitized using the WebPlotDigitizer software ${ }^{36}$ and reinvestigated using the new McChasy code.

The shape of the aligned spectrum recorded for the implanted sample does not reveal the characteristic damage peak that is a manifestation of the presence of point defects. Instead, the intensity of the spectrum increases gradually with decreasing energy. This is a signature of the presence of dislocation-like defects as the dominant type of defect formed in $\mathrm{Al}$ after the $\mathrm{Ni}$ implantation. The signal from the $\mathrm{Ni}$ is localized in the energy range from $\sim 1.4-1.55$ $\mathrm{MeV}$ in FIG. 5.

FIG. 5. RBS spectra recorded for Al single crystals using 2.0 MeV He ions moving along the [110] direction and detected at a backscattering angle of $160^{\circ}$. The Virgin (exp) aligned spectrum refers to the unimplanted sample, the Aligned (exp) and the Random (exp) spectra refer to the sample implanted at RT with $150 \mathrm{keV} \mathrm{Ni}$ ions to a fluence of $7 \times 10^{15} \mathrm{~cm}^{-2}$. 35 The data were digitized using the WebPlotDigitizer software. ${ }^{36}$ For the sake of clarity, the signal coming from $\mathrm{Ni}$ ions is magnified 16 times. The fits to the experimental data, denoted Random (sim), Virgin (sim), Aligned (sim1) and Aligned (sim2), were made using MC simulations in the new McChasy code. The Aligned spectra were fitted using dislocation parameters obtained for $\mathrm{SrTiO}_{3}(\operatorname{sim} 1)$ and $\mathrm{ZnO}(\operatorname{sim} 2)$.

Picraux et al. determined depth-distributions of Ni concentration from the RBS random spectrum and DIS concentration from the slope of the yield in the aligned spectrum. However, this method does not provide any physical units for the DIS concentration, so the term "relative disorder" was used instead. Both Ni and DIS profiles were digitized using the 
WebPlotDigitizer software ${ }^{36}$ and are reproduced in FIG. 6. The unit of DIS concentration was assigned later as described in the following paragraphs.

Based on the depth-distributions of Ni and DIS, MC simulations using the McChasy code were performed according to the following steps:

(i) First, the RBS random spectrum was fitted using the procedure of substituting target atoms by $\mathrm{Ni}$ impurities. Due to some disagreement between the $\mathrm{MC}$ simulation and the experimental spectrum, the Ni distribution was slightly adjusted as shown in FIG. 6. Afterward the aligned spectrum of the virgin sample was also fitted. The corresponding fits to the random and the virgin spectra are shown in FIG. 5.

(ii) $\mathrm{MC}$ simulations to the aligned spectrum of the implanted sample were performed taking into account the presence of dislocations only. The depth-distribution of DIS was obtained from Ref. 35, expressed in units $\mathrm{of}_{\mathrm{cm}}^{-2}$ and multiplied by different factors until a satisfactory fit was obtained (cf. FIG. 6 for the DIS concentration profile). Because dislocation parameters for $\mathrm{Al}$ are not known, those found for $\mathrm{SrTiO}_{3}$ were used instead, as both structures are cubic. The corresponding simulation of the aligned spectrum is shown in FIG. 5 (denoted $\operatorname{sim} 1)$.

(iii) To evaluate the importance of the dislocation parameters, simulations were repeated for the same DIS concentration using parameters of dislocations obtained for $\mathrm{ZnO}$. The outcome is shown in FIG. 5 as the short-dashed line denoted $\operatorname{sim} 2$.

FIG. 6. Depth-distributions of Ni (black triangles) and dislocations (circles) in $150 \mathrm{keV} \mathrm{Ni-implanted} \mathrm{Al}$ as taken from ${ }^{3,35}$ and digitized using the WebPlotDigitizer software. ${ }^{36}$ Corresponding distributions obtained from the new McChasy code are presented as horizontal step plots using solid gray and black lines, respectively.

The fit obtained by the McChasy code for the Ni-implanted Al aligned spectrum using the $\mathrm{SrTiO}_{3}$ parameters of dislocations ( $\left.\mathrm{sim} 1\right)$ is in very good agreement with the experimental 
data. The difference between the Aligned (exp) and the Aligned (sim1) spectra increasing at lower energies denotes that the actual dechanneling is stronger than it comes from simulations. This can be due to the non-optimised dislocation parameters used for the simulations but can also be influenced by the considered maximum depth of the defect profile.

Simulations repeated using the $\mathrm{ZnO}$ dislocation parameters (with all other parameters kept unchanged) gave a noticeably lower intensity ( $\operatorname{sim} 2)$. This observation indicates the high importance of the dislocation parameters used in the model. Therefore, the resultant DIS distribution for Ni-implanted $\mathrm{Al}$ cannot be considered as definitive until dislocation parameters for $\mathrm{Al}$ become known. Nevertheless, the shape of the spectrum is fairly reproduced using the present model of dislocations.

Furthermore, the Ni-implanted $\mathrm{Al}$ case shows that dislocations have a very strong impact on channeling spectra and their presence should not be neglected in the analysis of damage in crystals. However, for the case of materials revealing the presence of different types of defects in the same sample, the quantitative separation of their contribution to the backscattering yield is crucial but barely possible without computer simulations.

\section{B. Monte Carlo simulations for Er-implanted $\mathrm{ZnO}$}

FIG. 7 presents RBS spectra recorded for ZnO single crystals implanted at RT with $300 \mathrm{keV}$ Er ions reported in Ref. 37. Only a selected channeling spectrum referring to a sample implanted to a fluence of $2.0 \times 10^{-15} \mathrm{~cm}^{-2}$ as well as random and virgin spectra are shown. The shape of the channeling (aligned) spectrum indicates the formation of extended defects along with randomly displaced atoms. The latter gives rise to the damage peak visible in the energy range from $\sim 1.2-1.3 \mathrm{MeV}$, characteristic of point defects, defect clusters or amorphous regions. These kinds of defects give large contributions to direct backscattering. However, the high intensity of the spectrum behind the damage peak (below an energy of $1.2 \mathrm{MeV}$ ) 
indicates significant dechanneling of the beam, implying the presence of extended defects, e.g. dislocations. ${ }^{38}$ Indeed, e.g. TEM studies on $\mathrm{N}$-implanted $\mathrm{ZnO}$ revealed the presence of dislocation loops. ${ }^{39}$

FIG. 7. RBS spectra (symbols) recorded for $\mathrm{ZnO}$ single crystals using $1.7 \mathrm{MeV}$ He ions moving along the [0001] direction and detected at a backscattering angle of $170^{\circ}$. The Virgin spectrum refers to the unimplanted sample. The Random and the Aligned spectra refer to a sample implanted at RT with $300-\mathrm{keV} \mathrm{Er}$ ions to a fluence of $2.0 \times 10^{15} \mathrm{~cm}^{-2}{ }^{37}$ The fits to the experimental data (solid black lines) were obtained using $M C$ simulations using the new McChasy code. For the sake of clarity, the signal coming from Er ions is magnified 20 times.

The results of MC simulations are shown in FIG. 7 as solid black lines fitted to the experimental spectra. The McChasy code reproduced the channeling (aligned) spectrum based on separate depth-distributions of the RDA- and DIS-type defects. These defect profiles are shown in FIG. 8 as solid lines. The triangle symbols and the dash-dotted line (shown in FIG. 8 along with the RDA profiles) refer to the depth profile of $300 \mathrm{keV}$ Er ions in $\mathrm{ZnO}$ as determined directly from the experimental random spectrum and calculated by the McChasy code, respectively.

FIG. 8. Depth distributions of RDA and DIS in ZnO after implantation with $300 \mathrm{keV}$ Er ions to a fluence of $2.0 \times 10^{15} \mathrm{~cm}^{-2}$. The defect profiles are the result of $M C$ simulations using the McChasy code: those obtained using the 2-dimensional classical PSA approach are denoted as old (dashed lines) and were reported in Ref. 37, those obtained using the 3-dimensional updated PSA approach (described in §II.C.1) are denoted as new (solid lines). The corresponding simulated RBS/C spectra are shown in FIG. 7. The triangle symbols and the dash-dotted line on the upper plot are the depth profiles of Er-ions determined directly from the experimental random spectrum and calculated by the McChasy code, respectively (both referring to the right-hand vertical axis).

The results for the same sample reported in Ref. 37 are also presented for comparison in FIG. 8. Both simulations agree that the defect profiles of both types of defects are shifted relative to each other - dislocations are formed rather in deeper regions of the crystal than RDA with the peak concentration well beyond the region of maximum nuclear energy deposition. Although the general shapes of the defect profiles remain barely changed, some differences appear. The RDA profile fades out in a region located nearly $100 \mathrm{~nm}$ shallower than that calculated by the old version of the code. In contrast, the DIS profile indicates the presence of 
dislocations in slightly deeper regions and also postulates a concentration twice as high as for the previous simulations. All these observations are discussed in the following section.

\section{DISCUSSION}

The new McChasy code was used to fit RBS experimental spectra recorded for Al implanted with $150 \mathrm{keV} \mathrm{Ni}$ ions and for $\mathrm{ZnO}$ implanted with $300 \mathrm{keV}$ Er ions. The results obtained for Ni-implanted Al show good agreement with the experimental data. However, the dislocation parameters used for the simulations were obtained for different structures. The results are thus a strong indication of the importance of the dislocation parameters used in the model. As described in the experimental section, the parameters can be determined from HRTEM measurements. The procedure of fitting an arctan function to bent atomic rows adjusted to the dislocation is provided as a Microsoft Excel script available as supplementary software.

Although the DIS distribution for the $\mathrm{Al}$ case was initially determined analytically it must be pointed out that this is only possible if dislocations are the dominant type of defect in a structure. Moreover, the dislocation profile obtained was expressed as "relative disorder". Conversion into the real density of dislocations (expressed in $\mathrm{cm}^{-2}$ ) was possible using the McChasy code.

For materials containing a mixture of different types of defects, as in the case of Er-implanted $\mathrm{ZnO}$, the McChasy code can perform MC simulations based on separate distributions of RDA and DIS. During subsequent iterations channeling ions interact with target atoms that are either localized in their equilibrium positions (changed only by thermal vibrations) or shifted according to the appropriate model of defects (e.g. RDA or DIS). Depending on the nature of the defect, an interaction can lead either to direct backscattering or to dechanneling of the ion. The Er-implanted $\mathrm{ZnO}$ case was successfully reinvestigated for the selected Er-fluence of $2.0 \times 10^{15} \mathrm{~cm}^{-2}$. The results of the McChasy simulations are presented in §IV.B. The defect 
profiles obtained using the newest and the older version of the McChasy code reveal some differences that require explanation.

According to the new McChasy code, the RDA concentration is now lower behind its maximum; hence, its range profile is also narrower. This should be an effect of the more realistic 3D model of the interactions and determination of impact parameters. In the majority of cases, the impact parameters calculated in $3 \mathrm{D}$ are smaller so they lead to more intense dechanneling of ions, especially in the deeper parts of the crystal. As mentioned in §II.C.1 and shown in FIG. 2, the simulated backscattering yield is higher in the 3D case. Therefore, the new McChasy code now reproduces a comparable intensity of the experimental spectrum for a lower RDA concentration. The shallower range of RDA is also in agreement with other authors who postulate that in deeper regions of $\mathrm{ZnO}$ the formation of extended defects is more likely rather than the presence of stable point defects. ${ }^{39-41}$ Although the new shape of the RDA profile seems to be more consistent with the Er-profile, it should be noted that the measured defect profiles reach considerably into the sample than expected from the ballistics of nuclear interactions (e.g. calculated by the SRIM code). This reveals the high mobility of defects during the implantation and their diffusion towards the bulk.

Indeed, according to the MC simulations, extended defects (here modeled as edge dislocations) are formed in the deeper regions of $\mathrm{ZnO}$. This phenomenon was also observed in $\mathrm{N}$-implanted $\mathrm{ZnO},{ }^{39}$ Ar-bombarded $\mathrm{ZnO},{ }^{41}$ or ion-implanted $\mathrm{SiC}^{42}$ The mechanism of creation of extended defects in the deeper regions of a bombarded structure can be explained by differences in defect mobility. Interstitials are typically more mobile than vacancies. When diffusing towards the bulk they can cluster with other interstitials forming extended defects, e.g. dislocation loop platelets, which are more energetically favorable and less mobile than point defects. ${ }^{40,43}$ 
However, the concentration of dislocations seems to be twice as high as reported previously. This is an effect of the updated model of dislocations. In the newest McChasy code, the orientation of dislocations (associated with their Burgers vectors) is determined randomly using a rotation matrix with the 6-fold symmetry in hexagonal structures and the 4-fold symmetry in cubic structures. The dislocation lines around the simulation channel are distributed uniformly within the crystal depth. This approach provides a more realistic calculation of the dechanneling of ions and takes into account also the transparency effect. Previously, the contribution to dechanneling from bent atomic rows could accumulate leading to an overestimation of the scattering probability. Therefore, the backscattering yield was higher for the lower concentration of dislocations. The final settlement of this issue can be determined either by complex HRTEM investigations or by chemical etching. A comparison with MD simulations could also deliver useful information.

\section{CONCLUSIONS}

The main challenge for the analysis of channeling spectra is to separate the contributions to the backscattering yield from different types of defects. This complicated task can be partially fulfilled by the computational evaluation of the direct backscattering and the dechanneling fractions from the measured yield. ${ }^{4}$ Another approach relies on simulation codes using different models of lattice distortion. ${ }^{5-14}$ This paper describes improvements implemented in the Monte Carlo simulation software McChasy. The most recent changes include an updated model of dislocations and a unique model for 3D computation of ion-atom interactions. From now on the impact parameters of channeled ions as well as the thermal vibrations of target atoms are determined in $3 \mathrm{D}$.

The McChasy code has been used for a few decades to provide a better evaluation of RBS/C spectra than analytical methods. The use of atomic structures organized in atomic slices 
allows the calculation of deflections from more than one atom at a time. The improvements described in this paper make the code even more realistic and precise. However, some problems remain unsolved. Further development of the code will be focused e.g. on implementing dislocation loops, which are more likely created during implantation than the ideal edge dislocations. Nevertheless, the currently used model of dislocations seems to work well enough and along with the new 3D model for computation of ion-atom interactions, it makes the McChasy code one of the most powerful software tools available for the analysis of radiation damage in crystals by ion channeling.

\section{SUPPLEMENTARY MATERIAL}

See supplementary material for a selected arctan fit made using the Excel script described in $\S$ §III.

\section{ACKNOWLEDGMENTS}

This work was partially supported by the Polish Ministry of Science and Higher Education in the framework of the prize for young scientists awarded to the first author by the Institute of Electronic Materials Technology in Warsaw, Poland. We acknowledge support by the Fundação para a Ciência e Tecnologia (FCT) and FEDER grants PTDC/CTMCTM/28011/2017, LISBOA-01-0145-FEDER-028011, and UID/FIS/50010/2019. Parts of this research were possible thanks to the Ph.D. scholarship awarded to the first author by the National Centre for Nuclear Research in Otwock-Swierk, Poland. Special thanks to Dr. Nicholas Keeley from the National Centre for Nuclear Research in Otwock-Swierk, Poland, for linguistic corrections. 


\section{REFERENCES}

${ }^{1}$ A. Vantomme, Nucl. Instruments Methods Phys. Res. Sect. B Beam Interact. with Mater. Atoms 371, 12 (2016).

${ }^{2}$ E. Bøgh, Can. J. Phys. 46, 653 (1968).

${ }^{3}$ L.C. Feldman, J.W. Mayer, and S.T. Picraux, Materials Analysis by Ion Channeling: Submicron Crystallography (Academic Press, New York, 1982).

${ }^{4}$ K. Jin, G. Velisa, H. Xue, T. Yang, H. Bei, W.J. Weber, L. Wang, and Y. Zhang, J. Nucl. Mater. 517, 9 (2019).

${ }^{5}$ J.H. Barrett, Phys. Rev. B 3, 1527 (1971).

${ }^{6}$ O.S. Oen and M.T. Robinson, Nucl. Instruments Methods 132, 647 (1976).

${ }^{7}$ P.J.M. Smulders and D.O. Boerma, Nucl. Inst. Methods Phys. Res. B 29, 471 (1987).

${ }^{8}$ A. Dygo and A. Turos, Phys. Rev. B 40, 7704 (1989).

9 E. Albertazzi, M. Bianconi, G. Lulli, R. Nipoti, and M. Cantiano, Nucl. Instruments Methods Phys. Res. Sect. B Beam Interact. with Mater. Atoms 118, 128 (1996).

${ }^{10}$ G. Lulli, E. Albertazzi, M. Bianconi, G.G. Bentini, R. Nipoti, and R. Lotti, Nucl. Instruments Methods Phys. Res. Sect. B Beam Interact. with Mater. Atoms 170, 1 (2000).

${ }^{11}$ L. Nowicki, A. Turos, R. Ratajczak, A. Stonert, and F. Garrido, Nucl. Instruments Methods Phys. Res. Sect. B Beam Interact. with Mater. Atoms 240, 277 (2005).

${ }^{12}$ K. Gärtner, Nucl. Instruments Methods Phys. Res. Sect. B Beam Interact. with Mater. Atoms 227, 522 (2005).

${ }^{13}$ A. Turos, P. Jozwik, L. Nowicki, and N. Sathish, Nucl. Instruments Methods Phys. Res. Sect. B Beam Interact. with Mater. Atoms 332, 50 (2014).

${ }^{14}$ S. Zhang, K. Nordlund, F. Djurabekova, Y. Zhang, G. Velisa, and T.S. Wang, Phys. Rev. E 94, 043319 (2016).

${ }^{15}$ M.T. Robinson and I.M. Torrens, Phys. Rev. B 9, 5008 (1974). 
${ }^{16}$ B.T.M. Willis and A.W. Pryor, Thermal Vibrations in Crystallography (University Press, Cambridge, 1975).

17 J.F. Ziegler and J.P. Biersack, in Treatise Heavy-Ion Sci., edited by D.A. Bromley (Springer, Boston, MA, Boston, MA, 1985), pp. 93-129.

${ }^{18}$ L.R. Doolittle, Nucl. Inst. Methods Phys. Res. B 9, 344 (1985).

${ }^{19}$ J. Lindhard, Mat. Meddelelser K. Danske Vidensk. Selsk. 34, 1 (1965).

${ }^{20}$ A. Dygo and A. Turos, Phys. Lett. A 127, 281 (1988).

${ }^{21}$ A. Turos, R. Ratajczak, K. Pagowska, L. Nowicki, A. Stonert, and P. Caban, Acta Phys. Pol. A 120, 163 (2011).

${ }^{22}$ Z. Kopal, Numerical Analysis (Wiley, New York, 1961).

${ }^{23}$ A. Dygo and A. Turos, Radiat. Eff. 85, 237 (1985).

${ }^{24}$ A. Cromer, Am. J. Phys. 49, 455 (1981).

${ }^{25}$ P. Jozwik, N. Sathish, L. Nowicki, J. Jagielski, A. Turos, L. Kovarik, and B. Arey, Nucl. Instruments Methods Phys. Res. Sect. B Beam Interact. with Mater. Atoms 326, 234 (2014).

${ }^{26}$ M. Bianconi, E. Albertazzi, S. Balboni, L. Colombo, G. Lulli, and A. Satta, Nucl. Instruments Methods Phys. Res. Sect. B Beam Interact. with Mater. Atoms 230, 185 (2005).

${ }^{27}$ S. Creutzburg, E. Schmidt, P. Kutza, R. Loetzsch, I. Uschmann, A. Undisz, M. Rettenmayr, F. Gala, G. Zollo, A. Boulle, A. Debelle, and E. Wendler, Phys. Rev. B 99, 245205 (2019). ${ }^{28}$ R. Peierls, Proc. Phys. Soc. 52, 34 (1940).

${ }^{29}$ F.R.N. Nabarro, Proc. Phys. Soc. 59, 256 (1947).

${ }^{30}$ Y. Xiang, Commun. Comput. Phys. 1, 383 (2006).

31 P. Jóźwik, N. Sathish, L. Nowicki, J. Jagielski, A. Turos, L. Kovarik, B. Arey, S. Shutthanandan, W. Jiang, J. Dyczewski, and A. Barcz, Acta Phys. Pol. A 123, 828 (2013).

${ }^{32}$ L. Wieluński, D. Wieluńska, G. Della Mea, and A. Turos, Nucl. Instruments Methods 168, $323(1980)$. 
${ }^{33}$ J. Jagielski, A. Turos, L. Nowicki, P. Jozwik, S. Shutthanandan, Y. Zhang, N. Sathish, L. Thomé, A. Stonert, and I. Jozwik-Biala, Nucl. Instruments Methods Phys. Res. B 273, 91 (2012).

${ }^{34}$ P. Jóźwik, L. Nowicki, C. Mieszczyński, R. Ratajczak, A. Stonert, A. Turos, J. Jagielski, K. Lorenz, and E. Alves, in E-MRS 2018 Fall Meet. (Warsaw, 2018), p. J.5.2 (talk).

${ }^{35}$ S.T. Picraux, D.M. Follstaedt, P. Baeri, S.U. Campisano, G. Foti, and E. Rimini, Radiat. Eff. 49, 75 (1980).

${ }^{36}$ A. Rohatgi, WebPlotDigitizer 4.2, Https://Automeris.Io/WebPlotDigitizer (San Francisco, California, USA, 2019).

37 P. Jozwik, S. Magalhães, R. Ratajczak, C. Mieszczynski, M. Sequeira, A. Turos, R. Böttger, R. Heller, K. Lorenz, and E. Alves, Phys. Status Solidi Basic Res. 256, 1800364 (2018).

38 A. Turos, L. Nowicki, A. Stonert, K. Pagowska, J. Jagielski, and A. Muecklich, Nucl. Instruments Methods Phys. Res. Sect. B Beam Interact. with Mater. Atoms 268, 1718 (2010).

${ }^{39}$ G. Perillat-Merceroz, P. Gergaud, P. Marotel, S. Brochen, P.H. Jouneau, and G. Feuillet, J. Appl. Phys. 109, 023513 (2011).

${ }^{40}$ A. Azarov, P. Rauwel, A. Hallén, E. Monakhov, and B.G. Svensson, Appl. Phys. Lett. 110, 022103 (2017).

${ }^{41}$ A. Turos, P. Jóźwik, M. Wójcik, J. Gaca, R. Ratajczak, and A. Stonert, Acta Mater. 134, 249 (2017).

${ }^{42}$ J. Wong-Leung, M.S. Janson, A. Kuznetsov, B.G. Svensson, M.K. Linnarsson, A. Hallén, C. Jagadish, and D.J.H. Cockayne, Nucl. Instruments Methods Phys. Res. Sect. B Beam Interact. with Mater. Atoms 266, 1367 (2008).

${ }^{43}$ C.M. Wang, W. Jiang, W.J. Weber, and L.E. Thomas, J. Mater. Res. 17, 2945 (2002). 
(a)

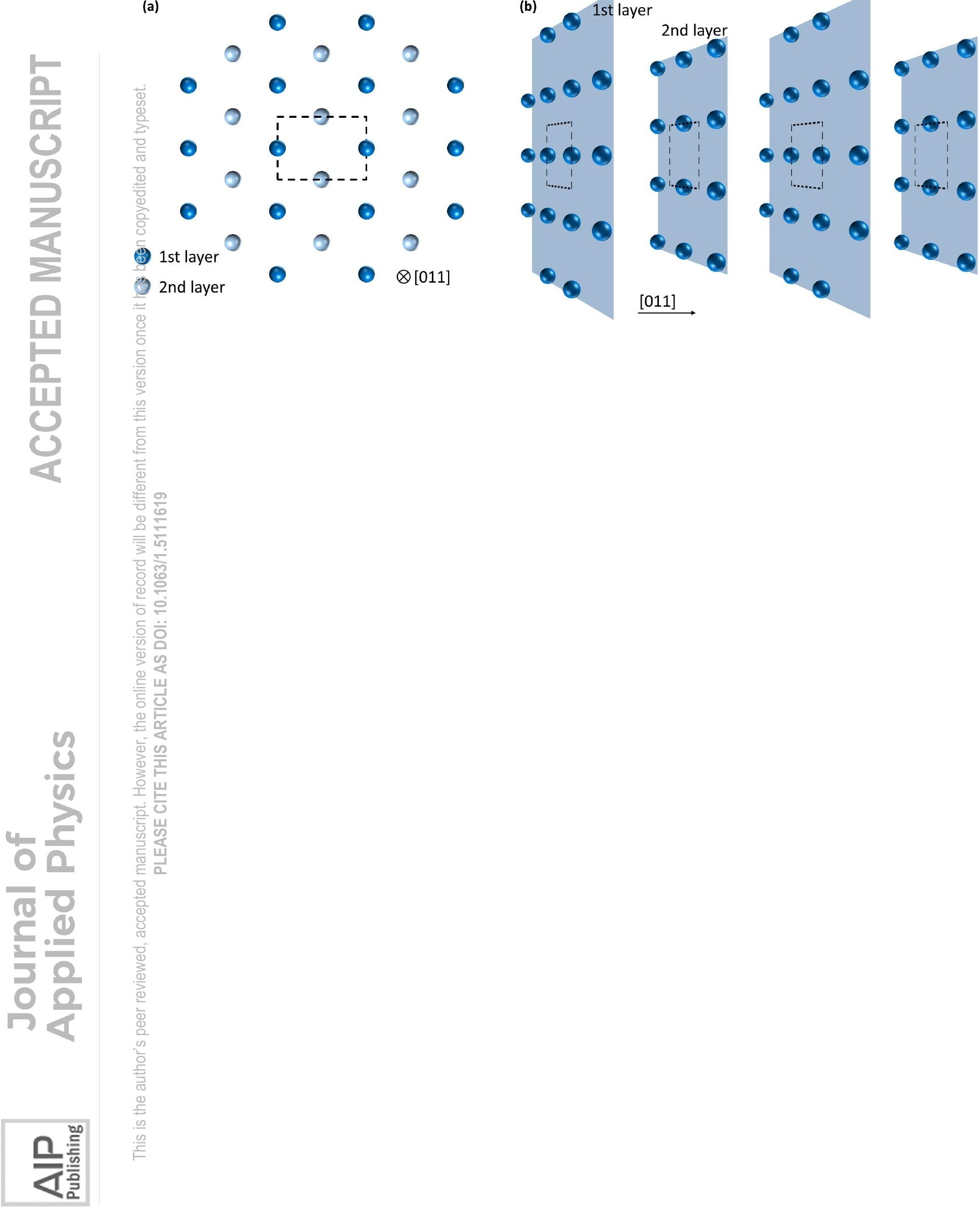



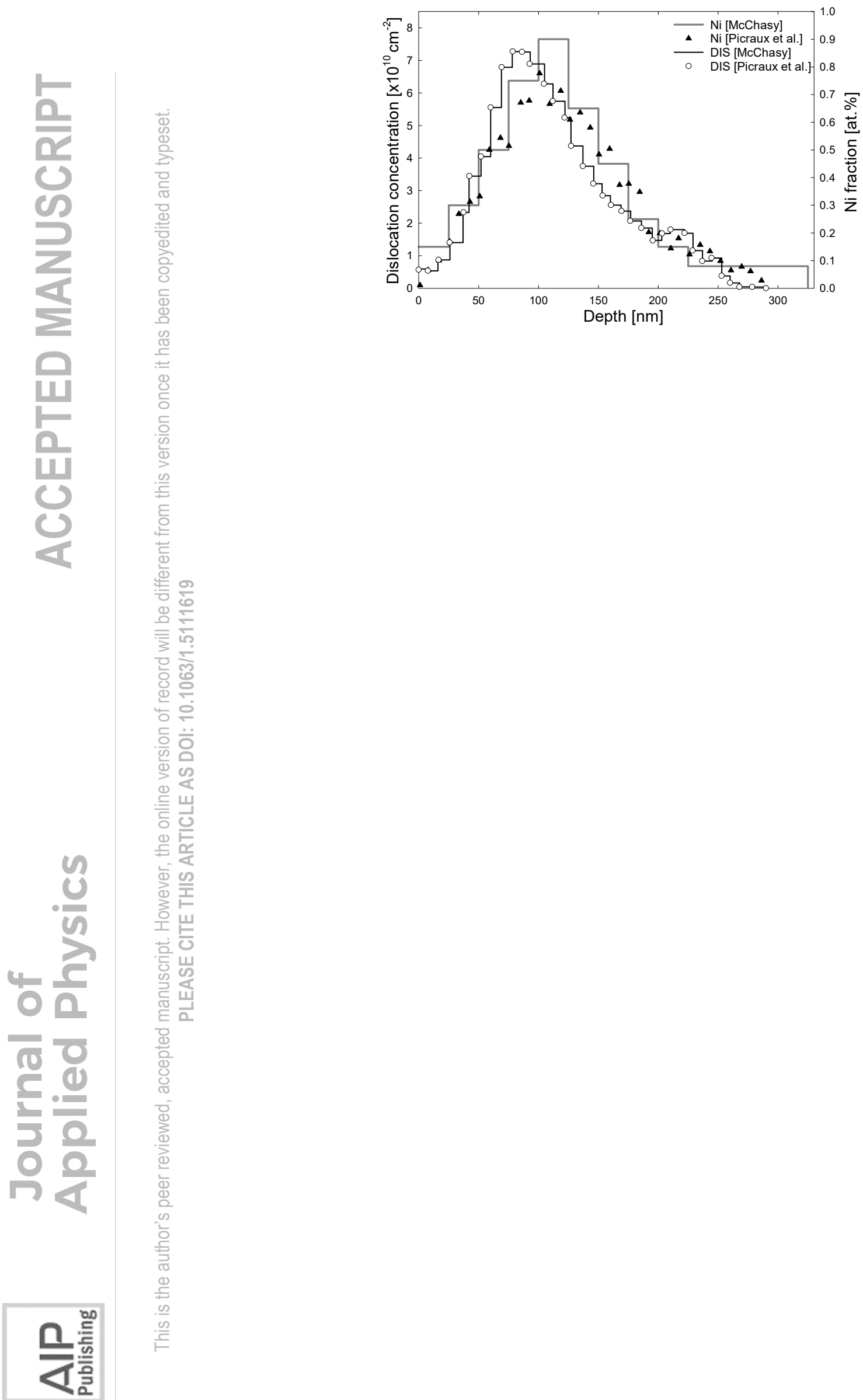

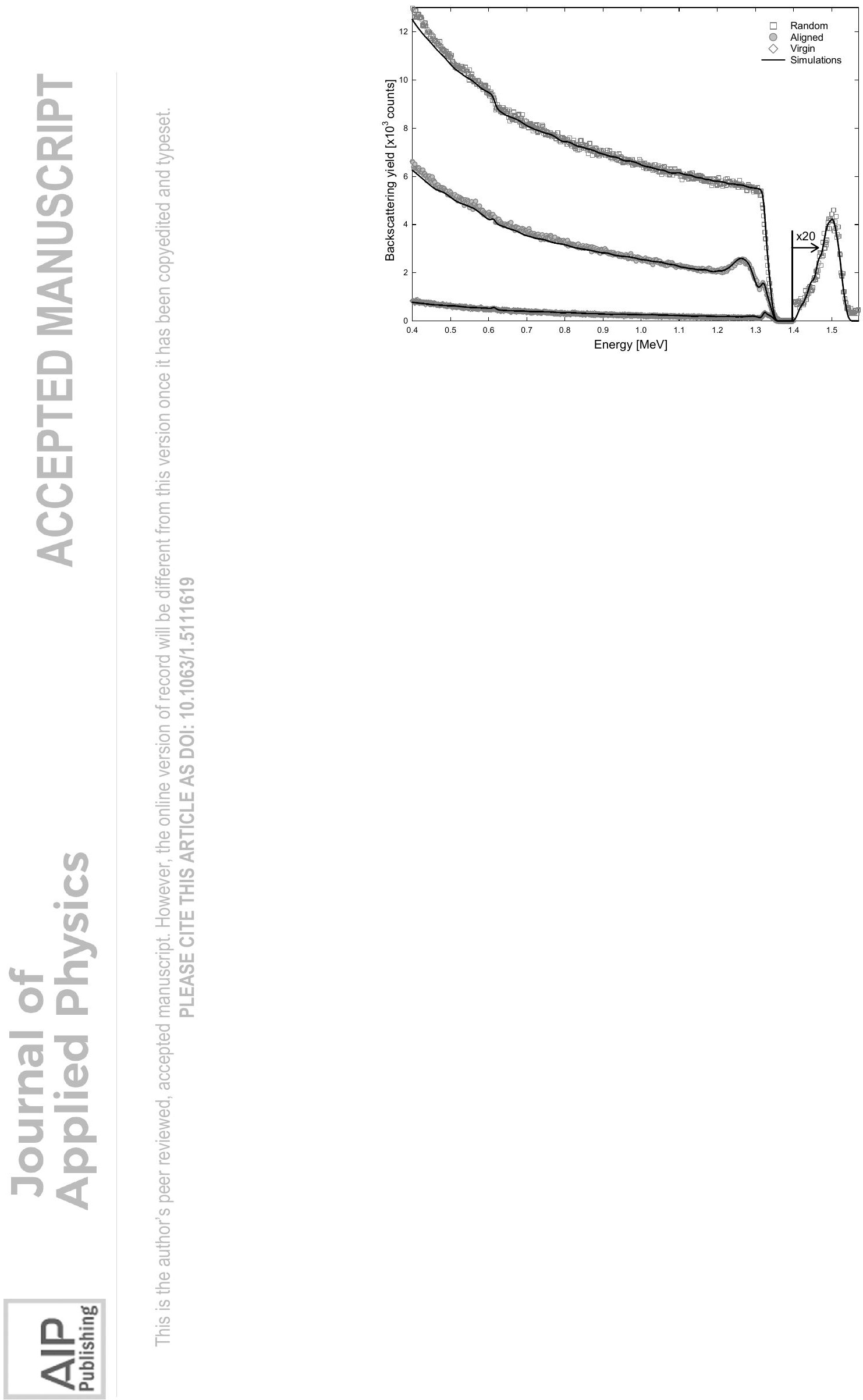
Depth [nm]

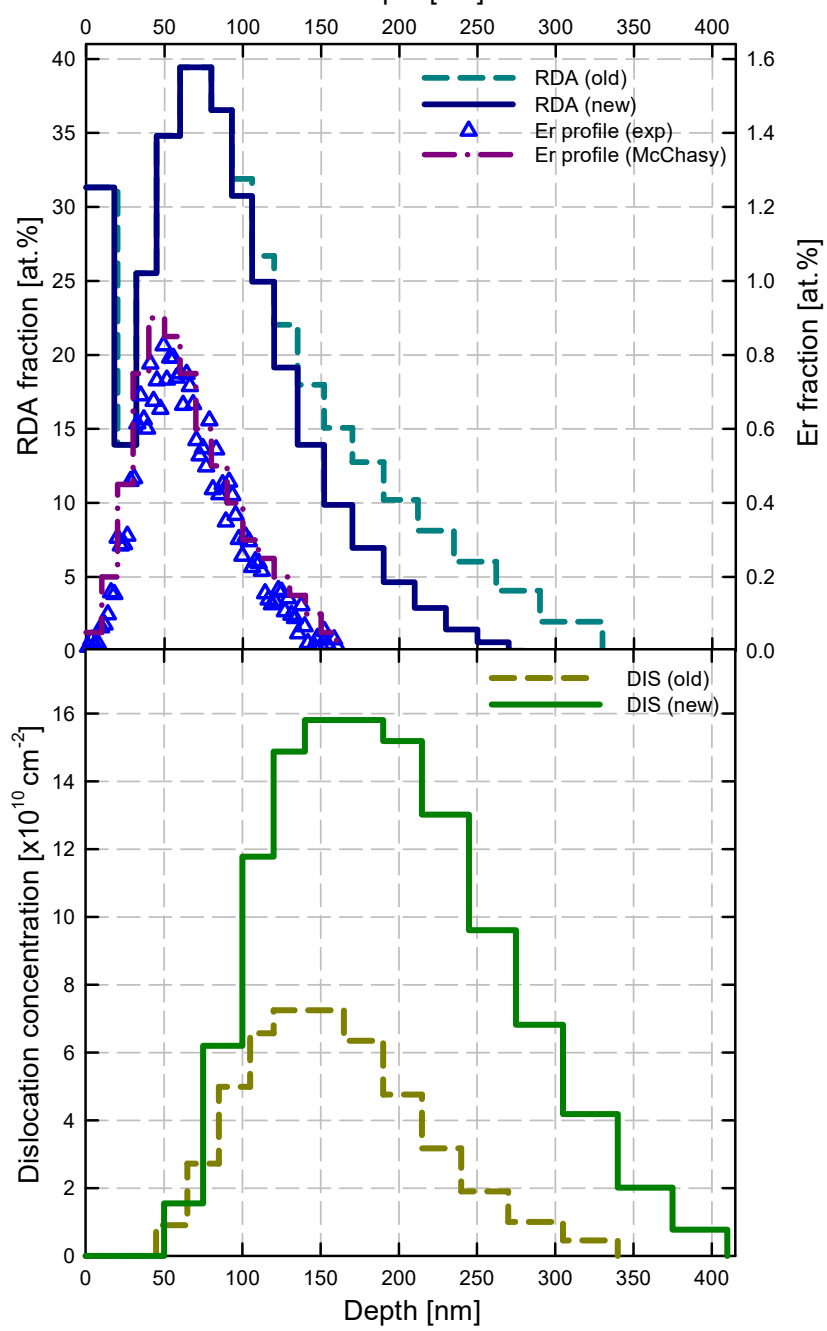

